\title{
Estudio del comportamiento del hormigón armado ante esfuerzos normales y tangentes mediante modelos seccionales de interacción completa
}

\author{
Behavior of reinforced concrete under normal and tangential \\ forces by means of Total-Interaction-Nonlinear-Sectional- \\ Analysis (TINSA)
}

J. M. Bairán ${ }^{(*)}$, A. R. Marí(*), S. Mohr ${ }^{(*)}$

RESUMEN

El análisis tradicional de estructuras reticulares se basa en las teorías clásicas de vigas cuyas hipótesis de partida implican la única existencia de tensiones normales en la sección transversal o, en algunos casos, también tensiones tangenciales con simplificaciones más o menos acertadas. Su aplicación resulta satisfactoria en casos dominados por solicitaciones normales. Existen varios intentos de extenderlos directamente al análisis no lineal de estructuras de hormigón con cargas arbitrarias donde dichas teorías no son aplicables estrictamente debido, entre otras cosas, a la fisuración diagonal. Así pues, la respuesta real ante solicitaciones importantes del tipo cortante, torsión o confinamiento, no puede ser reproducida adecuadamente por los métodos tradicionales. En este artículo se analizan las ventajas del análisis seccional y se define hasta qué punto considerar sólo el efecto de los esfuerzos normales puede resultar suficiente. Se presenta brevemente un modelo que permite reproducir estados tensionales tridimensionales a nivel seccional y se emplea para analizar estructuras sometidas a casos con notable influencia de esfuerzos tangenciales y confinamiento y se investiga la incidencia práctica en la respuesta de secciones y estructuras continuas. Se resalta la existencia de una distribución de tensiones normales modificada en la zona de tracciones que puede afectar la rigidez real de la estructura. Asimismo, se manifiesta un efecto dual al incremento de tracciones en la armadura longitudinal consistente en un incremento de tracciones en la armadura transversal con momento concomitante. Por otro lado, el modelo permite una representación más real de la fisuración en vigas y evidencia una mayor capacidad de rotación plástica antes de la rotura.

Palabras clave: Cortante, Torsión, Fisuración oblicua, Hormigón Armado, Análisis no lineal.

\section{SUMMARY}

Frame structural analysis has been traditionally based on classic beam theories whose starting hypotheses imply the existence of solely normal stresses in the cross-section or, in some cases, shear stresses in simplified fashion. These methods have been satisfactorily used in the nonlinear analysis of structures dominated by normal forces. Further, there have been many intends to directly extend them to non-linear analysis of concrete structure under more general loading where the theories cannot be applied in a general fashion due to the inclined cracking and anisotropic material behavior. Hence, real structural response under important shear, torsion or confinement cannot be suitably reproduced by traditional beam methods. In this paper, the advantages of frame element idealization of structures are analyzed and the applicability range of traditional schemes considering only normal stresses is defined. A sectional model capable of reproducing 3D stress states in the cross-section domain is briefly presented and used to analyze the response of sections and continuous structures under load cases influenced by tangential forces and confinement. It should be highlighted that the real distribution of normal stresses may be modified in the cracked zone of the beam and affect the structural stiffness. Hence, a shift in the stresses of transverse reinforcement under concomitant bending moments with shear is to be noted as the reciprocal manifestation of the well-known shift of stresses in the longitudinal reinforcements. On the other hand, the model allows a more realistic representation of actual shear patterns of beams and evaluation of actual rotation capacity on length of plastic regions.

\section{$451-15$}

Keywords: Shear, Torsion, Inclined cracking, Reinforced concrete, Nonlinear analysis.
Informes de la Construcción Vol. 62, 518, 65-77, abril-junio 2010 ISSN: 0020-0883 elSSN: 1988-3234 doi: 10.3989/ic.09.021

\footnotetext{
${ }^{(*}$ Departamento de Ingeniería de la Construcción. Universidad Politécnica de Cataluña (UPC) (España) Persona de contacto / Corresponding Author: jesus.miguel.bairan@upc.edu (J. M. Bairán)
}

Fecha de recepción: 02-06-09 Fecha de aceptación: 09-10-09 


\section{INTRODUCCIÓN}

El análisis del comportamiento de los sistemas estructurales puede abordarse mediante diferentes niveles de idealización, pasando desde la consideración del mismo como un sólido tridimensional, hasta su asimilación a un sistema reticular de barras caracterizadas por una sección transversal, tal como se representa en la Fig. 1.

Si bien la modelización como sólido puede ser más representativa de la realidad física de las estructuras de ingeniería, se debe reconocer que los modelos de barras presentan ventajas importantes respecto a aquélla. Aunque habitualmente se suele mencionar como principal ventaja el menor coste computacional de los modelos de barra, lo cierto es que ésta, aunque importante, va perdiendo relevancia con el desarrollo de software y hardware más eficientes. Actualmente, los principales aspectos que hacen que los modelos de barras sigan siendo, con diferencia, los más empleados para idealizar estructuras de ingeniería civil son:

- Facilidad en la construcción del modelo

- Interpretación de resultados en términos de esfuerzos generalizados directamente aplicable al dimensionamiento

- Reducción de grados de libertad del sistema

- Menor coste computacional

- Resultados muy satisfactorios para las regiones " $\mathrm{B}$ " gobernadas por esfuerzos normales.

Los modelos de barras se han aplicado al análisis no lineal de estructuras de forma satisfactoria, siendo capaces de reproducir numerosos fenómenos que tienen lugar en el hormigón armado, incluyendo el comportamiento postfisuración, próximo a la rotura, fenómenos diferidos, acciones ambientales, etc. Ver Marí (1), Marí y Bairán (2), entre otros. En este sentido, la respuesta de toda la barra viene caracterizada por la respuesta de la sección transversal. Por lo tanto, una adecuada simulación de la respuesta de la sección ante los esfuerzos a la que se ve sometida es trascendental en los resultados predichos.

A pesar de la versatilidad de los modelos de barras es necesario reconocer ciertas carencias implícitas en las formulaciones de los mismos. Concretamente, al considerar que los elementos son suficientemente largos, se desprecia la existencia de tensiones y deformaciones en direcciones diferentes a la normal a la sección, por lo tanto, sólo son capaces de reproducir los efectos de esfuerzos normales: axil y flexión. Asimismo, la geometría de la sección transversal es invariable con la solicitación y sólo es posible estudiar "regiones B". Si bien estas limitaciones no afectan a un número importante de casos prácticos, resulta necesario mejorar algunas de ellas para reproducir fenómenos de carga más generales en estructuras constituidas de materiales como el hormigón armado. Por ejemplo, los esfuerzos tangenciales (cortante y torsión) y los efectos del confinamiento producido por armadura transversal o por encamisado de chapa. En el contexto de este artículo, se denominarán "elementos fibra tradicionales" a los modelos seccionales con estas características descritas en este párrafo.

Algunos de los aspectos que pueden mejorarse son la existencia de un estado multiaxial de tensiones y deformaciones y la presencia de armadura transversal, cuya elongación implica la necesaria distorsión de la geometría de la sección transversal. Estas limitaciones hacen que exista un importante desequilibrio entre el nivel de precisión alcanzada para solicitaciones de esfuerzos normales puros respecto a los casos en que existen esfuerzos tangenciales. El interés de incluir estas mejoras en los modelos de barras abarca un gran número de aplicaciones: evaluación del comportamiento no lineal de estructuras de hormigón ante cargas estáticas que producen fuertes solicitaciones normales y tangentes, evaluación de la capacidad de redistribución real de las estructuras, estudio de estructuras de compuestos no-isótropos, etc.

Entre estas aplicaciones, cabe resaltar la gran relevancia en el estudio del comportamiento sísmico de las estructuras de hormigón, ya que en los grandes terremotos recientes los fallos estructurales en elementos supuestamente bien construidos de acuerdo a normativas modernas tienen involucradas, de una u otra forma, esfuerzos de cortante o torsión, ver Fig. 2. Por otro lado, se debe tener en cuenta que, frecuentemente, en el proyecto sismorresistente se espera la formación de zonas plásticas en los extremos de las vigas y pilares donde los esfuerzos cortantes, momentos flectores y esfuerzos axiles son máximos al mismo tiempo. Más aún, el rango natural de trabajo de estas regiones es el no lineal. Por lo tanto, la necesidad de disponer de modelos de barras capaces de reproducir satisfactoriamente el comportamiento de estas regiones es evidente.

En los últimos años se ha realizado un gran esfuerzo en el desarrollo de modelos seccionales capaces de dar solución a las necesidades arriba indicadas, Vecchio y Collins(3), Petrangeli (4), Ranzo (5), Bentz (6), entre otros. En general, estos modelos abordan el problema de flexión recta de secciones simétricas o bien consideran el estado de carga 
más general de forma simplificada mediante la discretización de la secciones en paneles con alguna hipótesis en la deformación de cortante de forma que no siempre se garantiza el equilibrio entre fibras. Por otro lado, en Bairán y Marí (7-9) se ha desarrollado un modelo seccional generalizado (TINSA) para estados de carga totalmente tridimensionales y secciones de geometría cualquiera. En Bairán y Marí (10) se hace un análisis de las diferentes metodologías existentes en la literatura y analiza la necesidad de incluir la distorsión y alabeo de la sección junto con una ecuación constitutiva tridimensional del hormigón para reproducir adecuadamente la respuesta para todos los niveles de carga.

En este artículo se analizan los aspectos fenomenológicos que tienen lugar en la interacción de esfuerzos normales y tangenciales y que dan lugar a acoplamiento de las tensiones en las armaduras longitudinales y transversales que, a pesar de ser observados en la experimentación, sólo se tienen en cuenta de forma parcial en el cálculo y dimensionamiento de estructuras de hormigón armado. Para ello se empleará el modelo seccional de interacción completa TINSA descrito en las referencias anteriores. El artículo parte de una breve descripción de los métodos de análisis seccionales tradicionales y de la problemática que deben abordarse para poder extenderlo hacia la consideración de esfuerzos tangenciales. Asimismo, presenta las ideas generales del modelo seccional de interacción completa empleado. Seguidamente se muestran los fenómenos de acoplamiento debidos a la acción simultánea de esfuerzos normales y tangentes y se discute la relevancia de los mismos.

\section{EL ANÁLISIS SECCIONAL MEDIANTE DISCRETIZACIÓN EN FIBRAS}

Uno de los métodos de análisis seccional más preciso consiste en la discretización en fibras de la geometría de la sección, Fig. 1b. Cada una de las fibras representa uno de los materiales constituyentes (p.e. hormigón o acero) y tiene en cuenta una ecuación constitutiva uniaxial. Dadas las deformaciones generalizadas de la sección, se pueden interpolar las deformaciones uniaxiales en cada una de las fibras mediante la ecuación de Navier-Bernoulli, Ec. 1. El procedimiento de análisis, descrito en la Fig. 3a, consiste en determinar la tensión en cada una de las fibras e integrar los esfuerzos generalizados. Este método permite obtener leyes de evolución tipo esfuerzo-deformación seccional como la que se muestra en la Fig. 3b reproduciendo todas las etapas de carga hasta la situación última.

$$
\varepsilon_{x}(z)=\varepsilon_{0}+z \phi_{y}-y \phi_{z}
$$
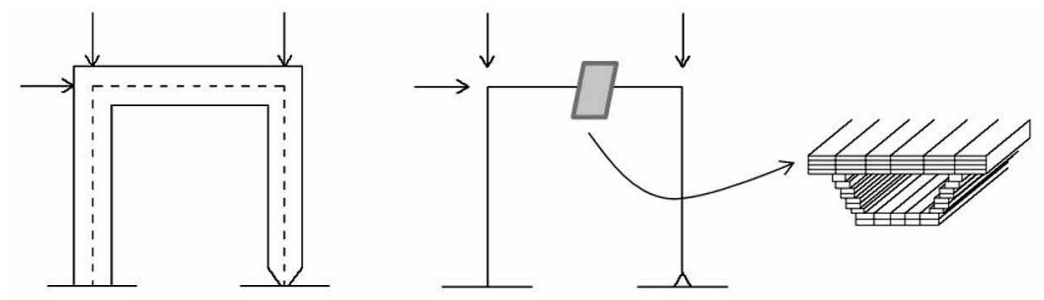

a)

b)
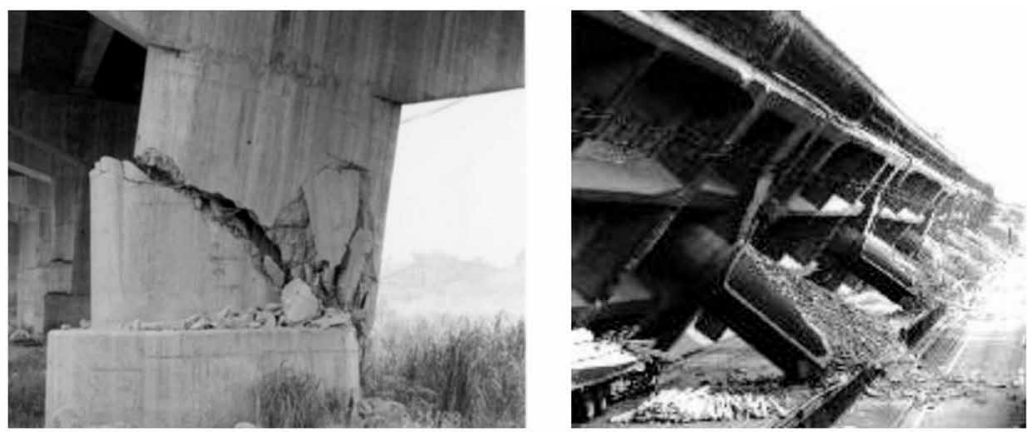

a)

b)
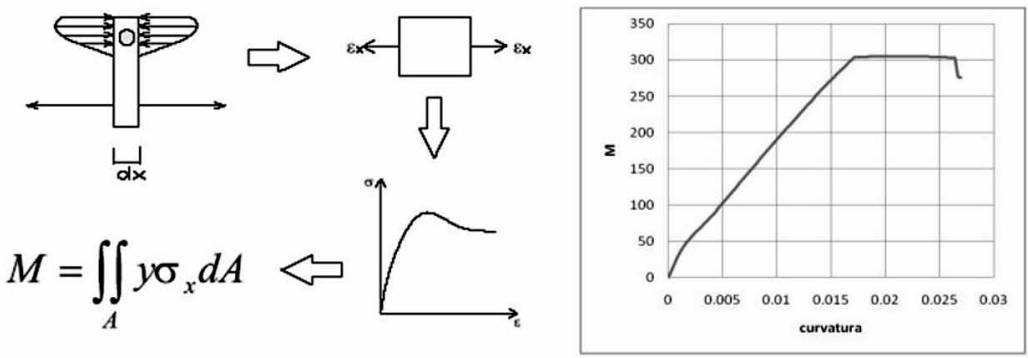

a)

b)

Por las propias hipótesis de partida, el esquema descrito sólo puede aplicarse en secciones con solicitaciones normales (axil y flexión). En el intento de extender el análisis para la consideración de esfuerzos tangentes es tentadora la idea de suponer una distribución de deformaciones de cortante en la sección, similar a la Ec. 1 para las deformaciones normales. Posteriormente, se evaluarían un estado de tensiones multiaxial en cada fibra y se integrarían los esfuerzos seccionales, que ahora incluirían el cortante y posiblemente la torsión. En la literatura reciente existen numerosos intentos de aplicar procedimientos similares, ver Bairán y Marí (10) para un análisis comparativo de algunos de ellos. Sin embargo, la aplicación de este tipo de metodologías en estructuras de hormigón fisuradas entraña ciertos errores de base que hacen que esta técnica sólo pueda aplicar-
1. Idealización estructural. a) Sólido 3D. b) Sistema reticular.

2. Roturas por cortante en terremotos recientes.

3. Integración de esfuerzos en sec ciones de fibras tradicional. a) Esquema del proceso. b) Diagrama momento-curvatura tipo. 

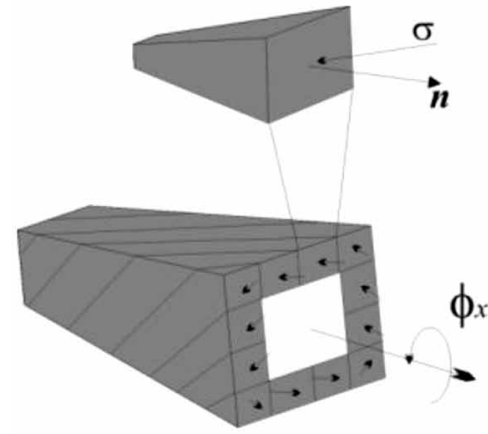

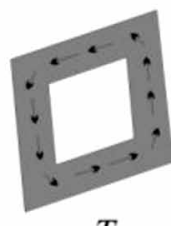

$T_{x}$
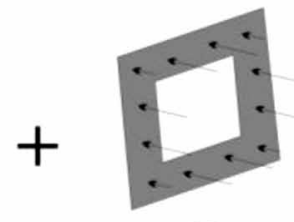

$N_{x}$

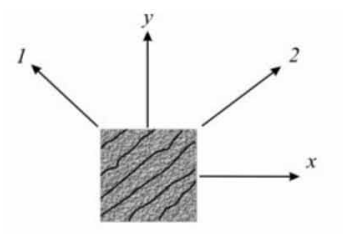

a)

4. Fisuración oblicua producida por esfuerzos tangenciales.

5. Fenómeno de anisotropía inducida por la fisuración. a) Interacción tensiones normales-cortantes. b) Una consecuencia práctica: interacción cortante-flexión.

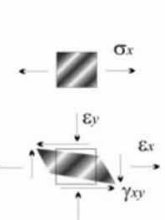

$M\left(\begin{array}{l}7 \\ v_{c} \\ \end{array}\right.$

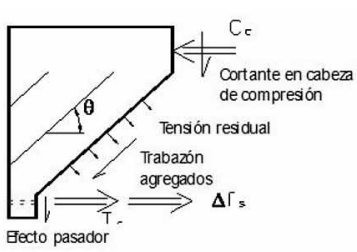

b)
4 armaduras longitudinales cuando existe un esfuerzo cortante concomitante. Este hecho se conoce y se tiene en cuenta, a efectos prácticos, mediante el "decalaje de la ley de momentos", como puede encontrarse en los libros de texto y las normativas vigentes de hormigón estructural, Jiménez Montoya et al (11), Park y Paulay (12), Eurocódigo EC2 (13), Instrucción de Hormigón Estructural EHE-08 (14), entre otros muchos. La interacción cortante-flexión puede explicarse mediante un corte oblicuo a través de la fisuración y estudiando el equilibrio del cuerpo libre resultante como se indica en la Fig. 5b o también haciendo un análisis plano de la sección transversal en el que se tiene en cuenta que el material es anisótropo como se muestra en Bairán (15).

No obstante, existe evidencia experimental, Hsu (16), Leonhard (17), Onsongo (18), Collins y Lampert (19), Cladera y Marí (20), entre otros, del acoplamiento que se produce entre los diversos esfuerzos, por ejemplo, axil-torsión, axil-cortante, flector-torsión, etc., sin que éstos sean tenidos en cuenta habitualmente. En general, podrán darse situaciones de acoplamiento completo dependiendo del patrón de fisuras generado.

En este punto, conviene definir el concepto de matriz de rigidez seccional como la matriz que relaciona el vector de esfuerzos de la sección transversal con las deformaciones de la viga en ese punto. En el caso de una barra de material isótropo cuyo eje de referencia pasa por el centro de cortantes la matriz de rigidez es de la forma que se muestra en la Fig. 6a. En este caso, los esfuerzos cortantes y de torsión están desacoplados del axil y los momentos flectores. Después de fisurar, la matriz de rigidez de una material no isótropo es, en términos generales, como se muestra en la Fig. $6 \mathrm{~b}$ donde todos los esfuerzos están acoplados. Se pone de manifiesto que, en virtud del conocido teorema reciprocidad de Maxwell, las matrices de rigidez llena debe ser simétrica y, por lo tanto, existirá un recíproco de cada fenómeno de acoplamiento. Así pues, el conocido efecto de interacción del cortante con la armadura longitudinal 


$\left[\begin{array}{c}N_{x} \\ V_{y} \\ V_{z} \\ T_{x} \\ M_{y} \\ M_{z}\end{array}\right]=\left[\begin{array}{cccccc}K_{11} & 0 & 0 & 0 & K_{15} & K_{16} \\ 0 & K_{22} & 0 & 0 & 0 & 0 \\ 0 & 0 & K_{33} & 0 & 0 & 0 \\ 0 & 0 & 0 & K_{44} & 0 & 0 \\ K_{51} & 0 & 0 & 0 & K_{55} & K_{56} \\ K_{61} & 0 & 0 & 0 & K_{65} & K_{66}\end{array}\right]\left[\begin{array}{c}\varepsilon_{0} \\ \gamma_{y} \\ \gamma_{z} \\ \phi_{x} \\ \phi_{y} \\ \phi_{z}\end{array}\right]$

a)

debe estar acompañado de su recíproco que produciría un incremento de tracción en la armadura transversal en virtud de un flector concomitante.

\section{GENERALIZACIÓN 3D. MODELO DE ANÁLISIS SECCIONAL CON INTERACCIÓN COMPLETA}

El análisis que se lleva a cabo en este artículo se realizará mediante un modelo de análisis no lineal de secciones que considera la interacción de esfuerzos axil, cortante, flexión y torsión desarrollado por Bairán (21). El modelo se conoce como TINSA ("Total Interaction Nonlinear Sectional Analysis") y ha sido aplicado satisfactoriamente a diversos problemas tridimensionales del hormigón armado y otros materiales compuestos, Bairán y Marí (7-10). Antes de explicar brevemente el modelo se debe reconocer, en primer lugar, la idea de que cualquier hipótesis cinemática a nivel sección que relacione las deformaciones de las fibras con las deformaciones generalizadas del elemento, como p.e. la Ec. 1, constituye una restricción artificial a la solución 3D de la estructura. Estas restricciones simplifican el problema real y facilitan una solución aproximada que será tan buena como la cinemática impuesta le permita.

En la Fig. 7 se representa la restricción de la hipótesis de sección plana como una recta en el espacio de soluciones 3D. La solución obtenida por la teoría de vigas basada en la deformación plana se representa por el punto indicado sobre la recta y constituye la solución más aproximada posible a la solución real, representada por la línea curva, que se puede conseguir sin violar la condición de sección plana. En la mayoría de los casos, el punto sobre la recta está suficientemente cerca a la solución real para aplicaciones prácticas. En otros, como pueden ser ciertas regiones $\mathrm{D}$, la aproximación no es satisfactoria y se reconoce que la teoría de vigas no

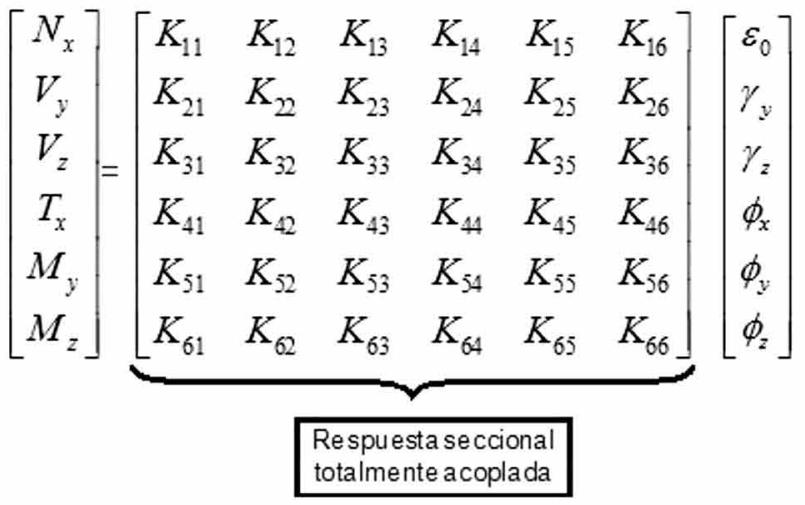

b)

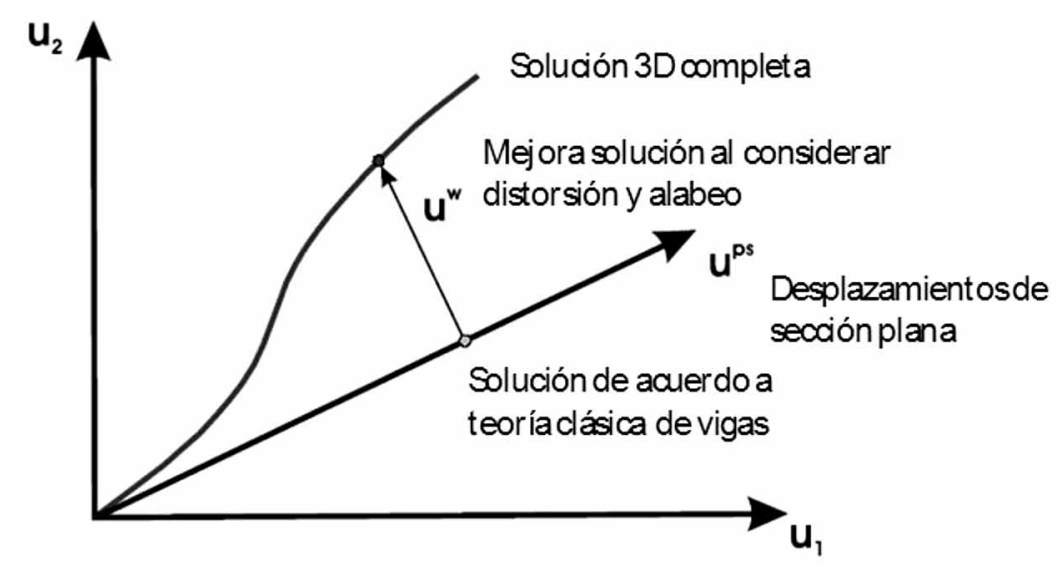

es aplicable ni siquiera en la situación lineal elástica. Esto es el caso, por ejemplo, de vigas de gran canto, zonas con aberturas, uniones viga-columna, etc. Por otro lado, ciñéndonos a aplicaciones de regiones $B$ de hormigón armado, existen estados 3D de tensiones y deformaciones que tienen lugar, tras la fisuración (máxime con fisuración inclinada) que no son compatibles con las hipótesis de sección plana. Así pues, las soluciones que ofrece la teoría tradicional de vigas son adecuadas solo en cierto rango de carga $y$, dependiendo del patrón de fisuras que se genera, pueden resultar no satisfactorias.

En TINSA se considera que la solución aproximada puede mejorarse, en principio, tanto como se quiera, sin necesidad de recurrir a un modelo totalmente 3D abandonando las ventajas de utilizar un modelo de vigas como se describió arriba. Esto se consigue añadiendo modos de deformación a la solución original que anteriormente no eran posibles, a base de alabeo y distorsión de la sección transversal. Esto se representa en la Fig. 7 mediante el vector $\mathbf{u}^{\mathrm{w}}$.

Se puede demostrar, Bairán y Marí (7), que las condiciones de equilibrio generales aplicables a cualquier hipótesis de viga están 
dadas por una única ecuación integral que debe satisfacer el elemento. En la ecuación 2 se muestra la relación en cuestión para situaciones de carga homogénea.

$$
\begin{aligned}
& R(x)=\iint_{A} \delta \boldsymbol{u}^{T} \boldsymbol{E}_{x}^{T} \boldsymbol{\sigma}^{\prime} d A- \\
& -\iint_{A} \boldsymbol{L}_{y z}(\delta \boldsymbol{u})^{T} \boldsymbol{\sigma} d A=0
\end{aligned}
$$

Donde $\sigma$ y $\sigma^{\prime}$ representan el tensor de tensiones $3 \mathrm{D}$ y su derivada en con respecto a la dirección del eje del elemento, dirección $x$. $\mathbf{E}_{x}$ es una matriz de términos ceros y unos definida en la ec. [3]. $L_{y z}$ es un operador diferencial lineal definido en la ec. [4].

$$
\begin{aligned}
\boldsymbol{E}_{x}{ }^{T} & =\left[\begin{array}{cccccc}
1 & 0 & 0 & 0 & 0 & 0 \\
0 & 0 & 0 & 1 & 0 & 0 \\
0 & 0 & 0 & 0 & 1 & 0
\end{array}\right] \\
\boldsymbol{L}_{y z}{ }^{T} & =\left[\begin{array}{cccccc}
0 & 0 & 0 & \frac{\partial}{\partial y} & \frac{\partial}{\partial z} & 0 \\
0 & \frac{\partial}{\partial y} & 0 & 0 & 0 & \frac{\partial}{\partial z} \\
0 & 0 & \frac{\partial}{\partial z} & 0 & 0 & \frac{\partial}{\partial y}
\end{array}\right]
\end{aligned}
$$

El término $\delta \mathbf{u}$ es un desplazamiento virtual compatible con la hipótesis cinemática predefinida. Por lo tanto, dependerá de la teoría de viga utilizada y es, además, el único componente que diferencia una teoría de viga respecto a otra cualquiera. Como puede verse en Bairán y Marí (10), sólo cambiando la forma de este vector en la expresión [2] pueden obtenerse diversas teorías de viga. En particular, si se sustituye la hipótesis 3D de Navier-Bernoulli, dada por la ecuación [5], se obtiene el sistema de ecuaciones [6] que coincide exactamente con el equilibrio de un elemento viga en el espacio.

$\boldsymbol{u}^{p s}=\left[\begin{array}{cccccc}1 & 0 & 0 & 0 & z & -y \\ 0 & 1 & 0 & -z & 0 & 0 \\ 0 & 0 & 1 & y & 0 & 0\end{array}\right]\left[\begin{array}{c}u_{0} \\ v_{0} \\ w_{0} \\ \theta_{x} \\ \theta_{y} \\ \theta_{z}\end{array}\right]$

$\iint_{A}\left[\begin{array}{c}\sigma_{x}{ }^{\prime} \\ \tau_{x y}{ }^{\prime} \\ \tau_{x z}{ }^{\prime} \\ -z \tau_{x y}^{\prime}+y \tau_{x z}{ }^{\prime} \\ z \sigma_{x}{ }^{\prime}, \\ -y \sigma_{x}{ }^{\prime}\end{array}\right] d A-$

$-\iint_{A}\left[\begin{array}{c}0 \\ 0 \\ 0 \\ 0 \\ \tau_{x z} \\ -\tau_{x y}\end{array}\right] d A=\left[\begin{array}{c}N_{x}{ }^{\prime} \\ V_{y}{ }^{\prime} \\ V_{z}{ }^{\prime} \\ T_{x}{ }^{\prime} \\ M_{y}{ }^{\prime} \\ M_{z}{ }^{\prime}\end{array}\right]-\left[\begin{array}{c}0 \\ 0 \\ 0 \\ 0 \\ V_{z} \\ -V_{y}\end{array}\right]=\left[\begin{array}{l}0 \\ 0 \\ 0 \\ 0 \\ 0 \\ 0\end{array}\right]$

La mejora a la teoría de vigas de NavierBernoulli se consigue sumando un campo de alabeo y distorsión $\left(\mathbf{u}^{w}\right)$ a los desplazamientos de la ecuación [5]; de esta forma, el campo de desplazamientos total de la sección transversal queda definido por la ecuación [7].

$\boldsymbol{u}=\boldsymbol{u}^{p s}+\boldsymbol{u}^{w}$

Como resultado, al sustituir [7] en [2], se obtiene un sistema de equilibrio dual. Uno de ellos representa el equilibrio de secciones planas de un elemento diferencial de viga, Fib. 8a. Estas condiciones de equilibrio son la base del sistema de elementos barras y se satisfacen a nivel estructura. Por otro lado, están las condiciones de equilibrio que garantizan que las secciones se mantienen íntegras asegurando que las fibras están en equilibrio respecto a las adyacentes, Fig. 8b. Este sistema de equilibrio se puede resolver internamente a nivel de la sección transversal.

Los modos de alabeo y distorsión pueden ser tan generales como se quiera $y$, en principio, podrían igualar la solución exacta, es decir, se estaría reproduciendo un modelo 3D. En el problema que nos ocupa, el interés es mejorar las soluciones en regiones $B$ para considerar fenómenos no lineales que tienen lugar en elementos alargados. Así pues, se admite la hipótesis de que la variación del alabeo y la distorsión a lo largo del eje del elemento es despreciable. Por lo tanto, el nuevo campo de desplazamientos no producirá tensiones normales a la sección. Sin embargo, es capaz de producir tensiones de cortante y normales fuera del plano de la sección transversal. Estas condiciones cinemáticas son suficientes para reproducir el estado tensión y deformación 3D que tiene lugar en los elementos largos con fisuración inclinada sin entrar en la modelización de regiones $\mathrm{D}$.

Esta formulación puede aplicarse juntamente con cualquier ecuación constitutiva multiaxial que considere el material como un continuo. En particular, para la simulación del hormigón fisurado resultan convenientes las ecuaciones constitutivas de fisuración distribuidas como la descrita en Bairán y Marí (10) o por la "Teoría Modificada del Campo de Compresiones" (3) en cualquiera de sus versiones.

\section{CASOS DE ESTUDIO}

A continuación se presentan una serie de ejemplos de aplicación a problemas de hormigón armado con la finalidad de evaluar la capacidad del modelo para simular problemas de interacción de esfuerzos normales y tangentes. Asimismo, se pretende resaltar la fenomenología que tiene lugar a nivel de distribución de tensiones en el hormigón y las armaduras indicando aquellas que se tienen en cuenta actualmente en el proyecto mediante consideraciones normativas. 


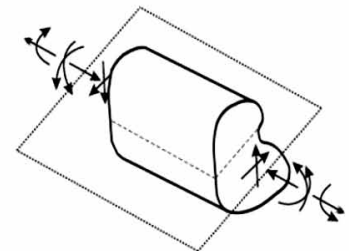

a)

\subsection{Interacción flexión-cortante en la respuesta de secciones}

El modelo presentado ha sido validado par diferentes combinaciones de carga tridimensionales y configuración de armado, ver Bairán y Marí (8-10). No obstante, a continuación se presenta la simulación de un caso experimental, Kani (22), de cortante puro a modo de validación. Dicho estado de carga se consigue aplicar mediante el esquema de ensayo representado en la Fig. 9 donde se ha puede observar que se consigue una sección con cortante y momento nulo durante todo el proceso de carga. La sección transversal de la viga y las propiedades mecánicas de los materiales se indican en la Fig. 10.

Por otro lado, la respuesta cortante-deformación de cortante de la sección se representa en la Fig. 11 juntamente con el resultado numérico predicho por el modelo seccional donde se observa una muy buena correlación en todos los niveles de carga.

A continuación se muestra un estudio sobre efecto del cortante en los diagramas momento-curvatura y del efecto dual de momento concomitante en la curva cortantedeformación angular. El análisis se hace sobre la misma sección que la Fig. 10. Los diagramas momento-curvatura obtenidos para cuatro relaciones $\mathrm{M} / \mathrm{V}$ diferentes se muestran en la Fig. 12. La influencia del cortante concomitante empieza a ser significativo para relaciones $M / V$ menores a 4

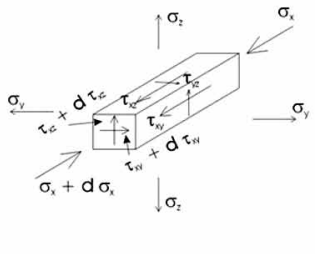

Leyde momentos:

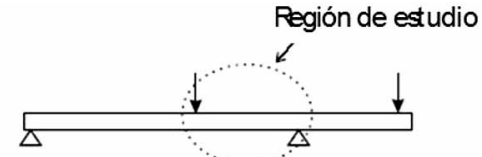

Ley de $\infty r t a n t e s:$
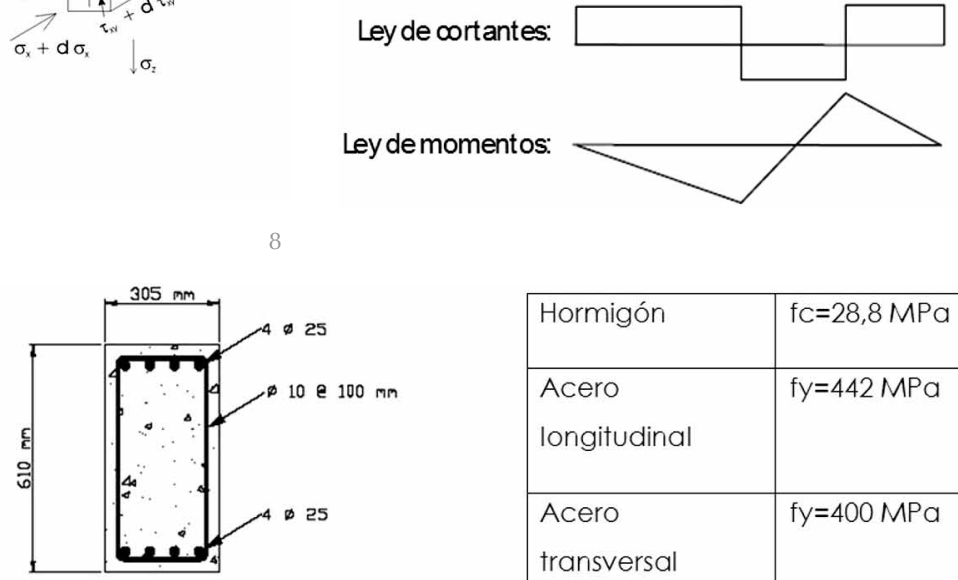

a)

\begin{tabular}{|l|l|}
\hline Hormigón & $\mathrm{fc}=28,8 \mathrm{MPa}$ \\
\hline $\begin{array}{l}\text { Acero } \\
\text { longitudinal }\end{array}$ & $\mathrm{fy}=442 \mathrm{MPa}$ \\
\hline $\begin{array}{l}\text { Acero } \\
\text { transversal }\end{array}$ & $\mathrm{fy}=400 \mathrm{MPa}$ \\
\hline
\end{tabular}

b)

d (canto útil). Las principales afectaciones en la respuesta a flexión consisten en una reducción de la rigidez fisurada para estos cortantes grandes.

Por otro lado, el punto de plastificación se reduce, así como la ductilidad en curvatura que puede alcanzar la sección antes de romper. Sin embargo, la pendiente de la rama plástica aumenta a medida que el cortante es mayor. Más adelante se mostrará que este último aspecto consigue que las curvaturas en una rótula plástica se distribuyan en una región más amplia; por lo tanto, se consiguen mayores rotaciones plásticas.

La Fig. 13, pág. siguiente, muestra la variación de la deformación en la armadura longitudinal con el momento aplicado para diferentes relaciones M/V. No se aprecia una variación importante en la deformación de las armaduras longitudinales para las relaciones de $4 d$ y $5 d$, sin embargo para cortantes superiores
8. Sistema de equilibrio dual. a) Nivel estructura. b) Nivel sección.

9. Esquema estático de un ensayo a cortante puro.

10. Sección transversal y propiedades mecánicas.

11. Comparación experimental-numérica curva cortante-deformación de cortante.

12. Diagrama momento-curvatura para distintos cortantes.

\section{Cortante-deformación de cortante}

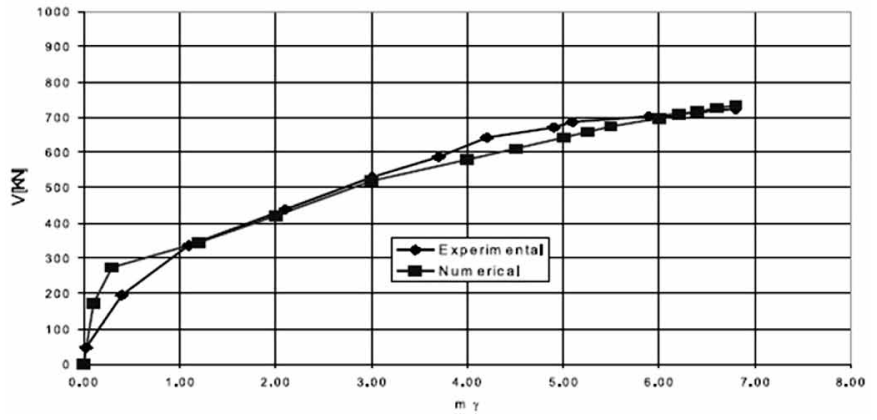

Momento- aurvaturapara distintas relaciones M/V

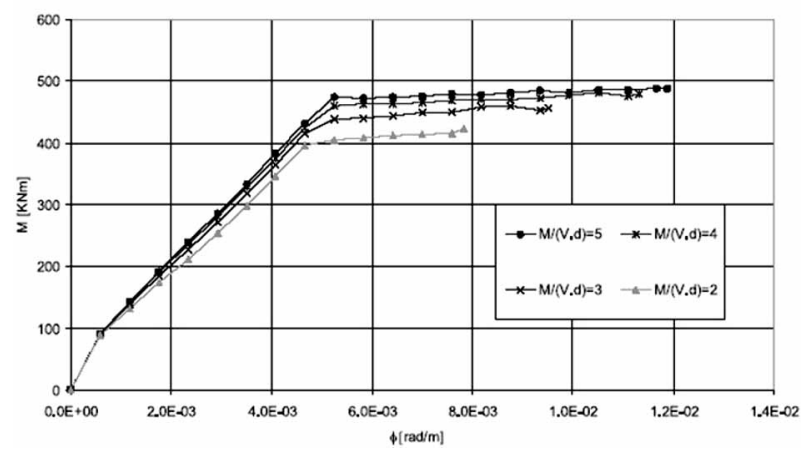


$\mathrm{M}$ - clong variando $\mathrm{M} / \mathrm{V}$

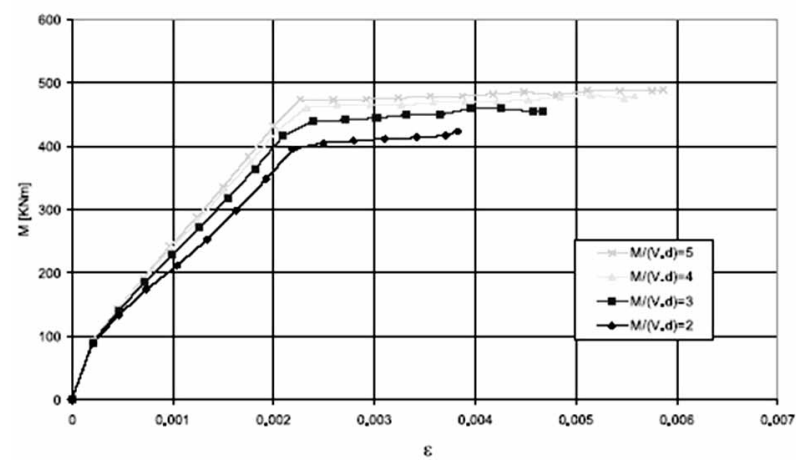

$\mathrm{V}$ - Etrans variando $\mathrm{M} / \mathrm{V}$

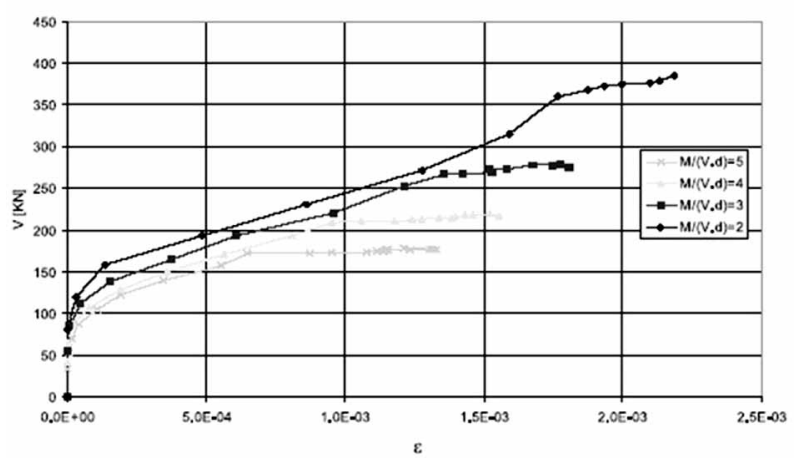

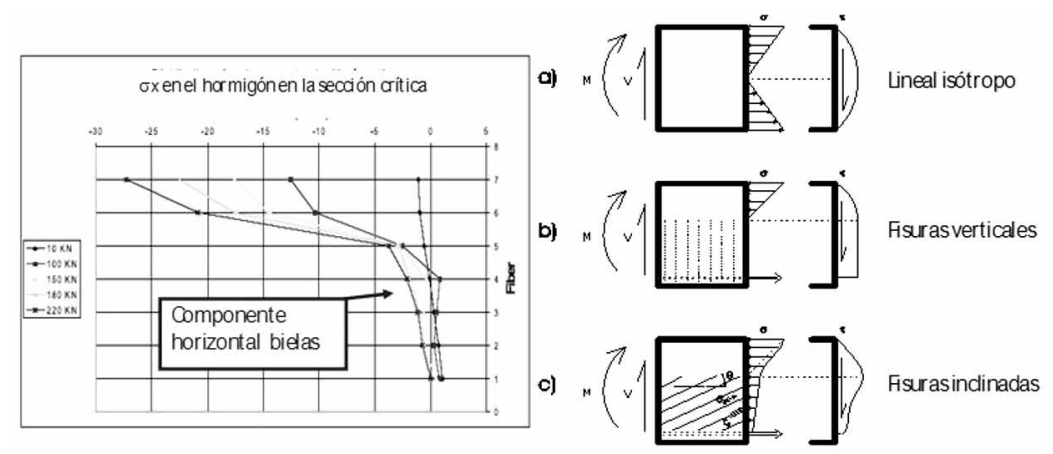

13. Diagrama momento-deformación armadura longitudinal para distintos cortantes.

14. Diagrama Cortante-deformación de cortante para distintos momentos

15. Estudio de la distribución de tensiones normales en una sección con fisuración inclinada.

16. Esquema estático de viga continua estudiada.

17. Secciones transversales de los modelos estudiados.
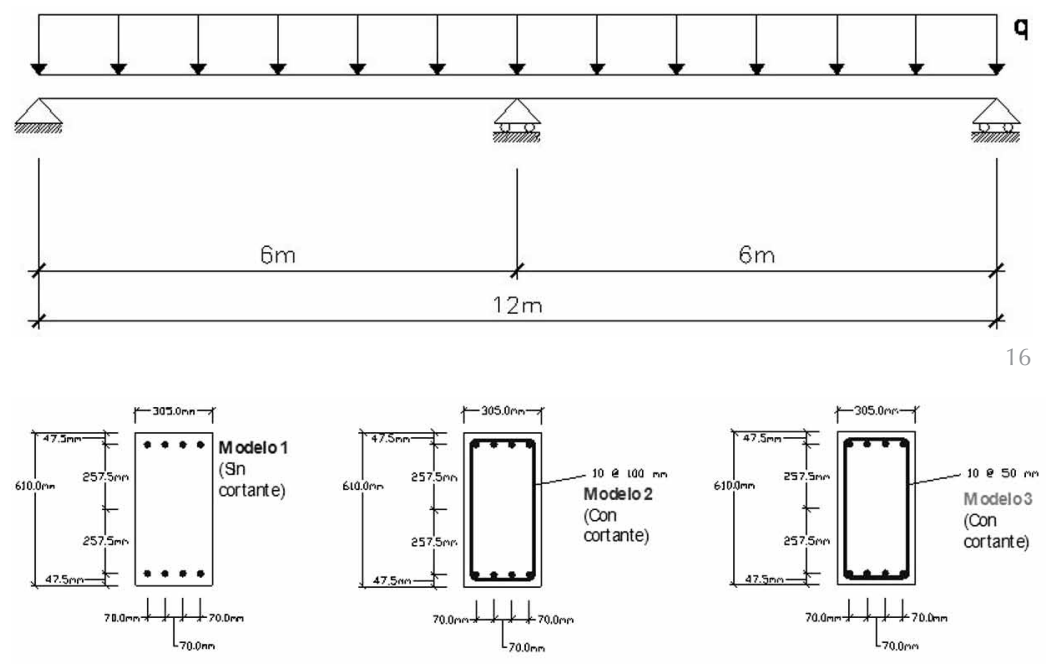

medida que aumenta la relación $M / V$, es decir, el momento concomitante es superior.

El fenómeno de fisuración inclinada en el alma de una sección de hormigón armado produce un efecto de campos de compresiones inclinadas que tiene una componente normal al plano de la sección como se aprecia en la Fig. 15. La integral de estas compresiones debe ser equilibrada por la armadura longitudinal de tracción, por un lado, y por una reducción de las compresiones en la cabeza superior.

\section{2. interacción flexión-cortante a nivel estructura}

Con la finalidad de investigar la influencia a nivel de estructuras completas de los fenómenos de interacción de esfuerzos normales y cortantes se plantea el estudio de la estructura continua representada en la figura 16. Sobre este esquema estático se analizarán los tres modelos de la Fig. 17. El Modelo 1 se trata de un análisis no lineal de vigas tradicional, es decir, sin considerar los efectos del cortante. Este modelo servirá de referencia para comparar los resultados. La armadura de este modelo consta exclusivamente de armaduras longitudinales que consisten en 4 ф $25 \mathrm{~mm}$ en ambas caras. Los elementos de viga utilizados son del tipo Navier-Bernoulli con dos puntos de integración.

Adicionalmente, se estudian los modelos denominados Modelo 2 y Modelo 3. Ambos modelos tienen la misma armadura longitudinal que el Modelo 1; por lo tanto, la única diferencia entre ellos es la armadura transversal siendo la cuantía transversal en el Modelo 3 el doble que en el 2. La armadura transversal es uniforme en toda la viga y los elementos finitos son de tipo Timoshenko con interpolación lineal y un único punto de integración en el centro del mismo.

La Fig. 18 muestra la curva carga-desplazamiento para cada uno de los modelos 


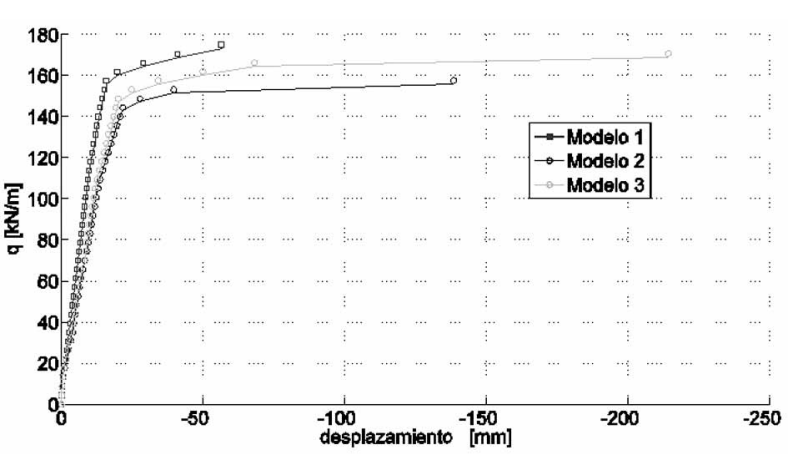

18

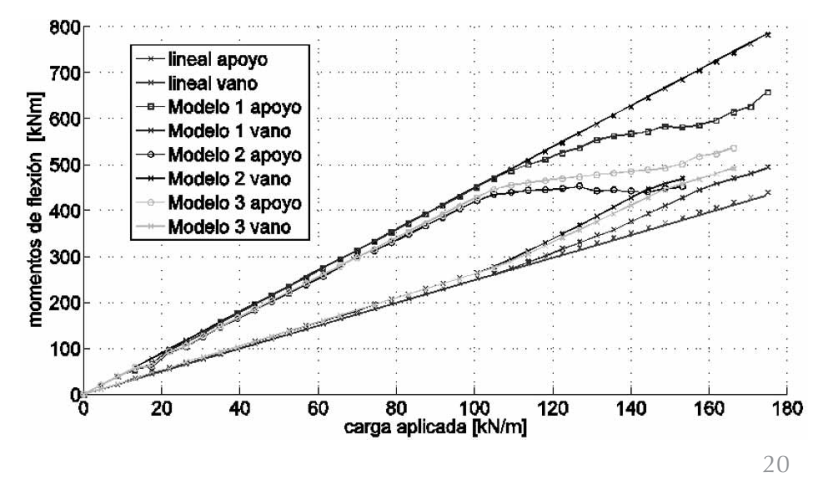

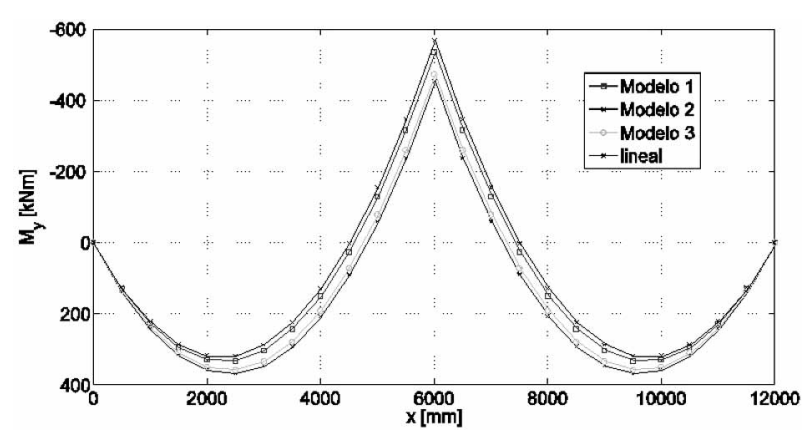

19

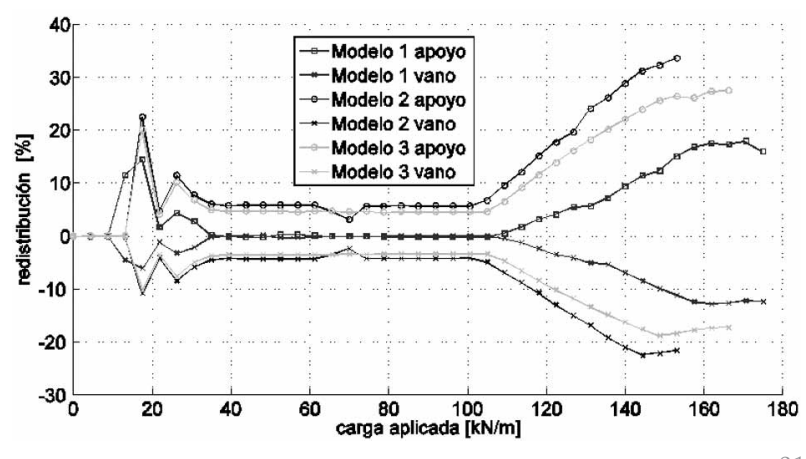

estudiados. Se observa, en primer lugar, que el modelo sin cortante (Modelo 1) alcanza una deformación inferior debido a que este desprecia la contribución del cortante a la deformación total. Las diferencias en la deformabilidad de la estructura se aprecian desde el momento en que ocurre la fisuración, ya que al no contemplar la fisuración por cortante resulta una estructura más rígida. La contribución de la fisuración a cortante en la flecha total es relativamente pequeña o moderada para cargas de servicio en función del armado dispuesto. En estudios sobre la deformabilidad a cortante, realizados por Tagliano (23), de carácter experimental, y por Lloveras (24), de carácter numérico, se ha estimado que ésta puede representar entre un $10 \%$ a $30 \%$ de incremento de la flecha instantánea.

No obstante, en el rango claramente no lineal, el aumento de flecha por deformación a cortante es más importante. A niveles de carga próximos al estado límite último este efecto es beneficioso ya que permite mayor capacidad de deformación y rotación y, por lo tanto, se consigue mayor redistribución de esfuerzos. La Fig. 19 muestra las leyes de momentos para un nivel de carga avanzado donde también se ha representado, a efectos de comparación, la ley de momentos lineal elástica. Se aprecia claramente que la redistribución conseguida es menor para el modelo que no considera cortante. Entre los modelos 2 y 3 la mayor redistribución se alcanza en el caso de menor cuantía. Esto se explica porque dicho modelo posee una longitud plástica superior. No obstante, el elemento con menor cuantía transversal alcanza una carga última menor ya que alcanza la plastificación de las armaduras transversales, como se verá más adelante.

La Fig. 20 muestra la evolución del momento en el centro de vano y en el apoyo para cada uno de los modelos y se compara con los valores de la ley lineal elástica. La diferencia para cada nivel de carga entre el momento lineal elástico y el realmente obtenido representa la redistribución de momentos alcanzada en cada sección. Puede observarse que en el caso del Modelo 2 se alcanza la redistribución completa formándose un mecanismo (momentos en apoyo y vano se igualan) aproximadamente para $\mathrm{q}=150 \mathrm{kN} / \mathrm{m}$. El Modelo 3 presenta menor incremento de tracciones en la armadura longitudinal y logra alcanzar una carga superior. Lo mismo ocurre con el Modelo 1 al no tener en cuenta el efecto del cortante.

El porcentaje de redistribución alcanzado en cada sección se cuantifica y representa en la Figura 21. Se aprecia que el elemento sin armadura transversal no alcanza el 20\% de redistribución, mientras que los modelos que contemplan el cortante sobrepasan el 25\% y el $30 \%$ en la sección de centro vano.

En la Fig. 22 se indica la distribución de tensiones en la armadura transversal en la mitad del canto de la viga para los casos Modelo 2 y Modelo 3. Es interesante hacer notar que ésta no sigue una distribución
18. Curva carga distribuida-desplazamiento para la viga continua.

19. Leyes de momento para $q=127$ $\mathrm{Kn} / \mathrm{m}$.

20. Relación de momentos en centro luz y en vano contra carga aplicada.

21. Porcentaje de redistribución de momentos en función de la carga aplicada. 

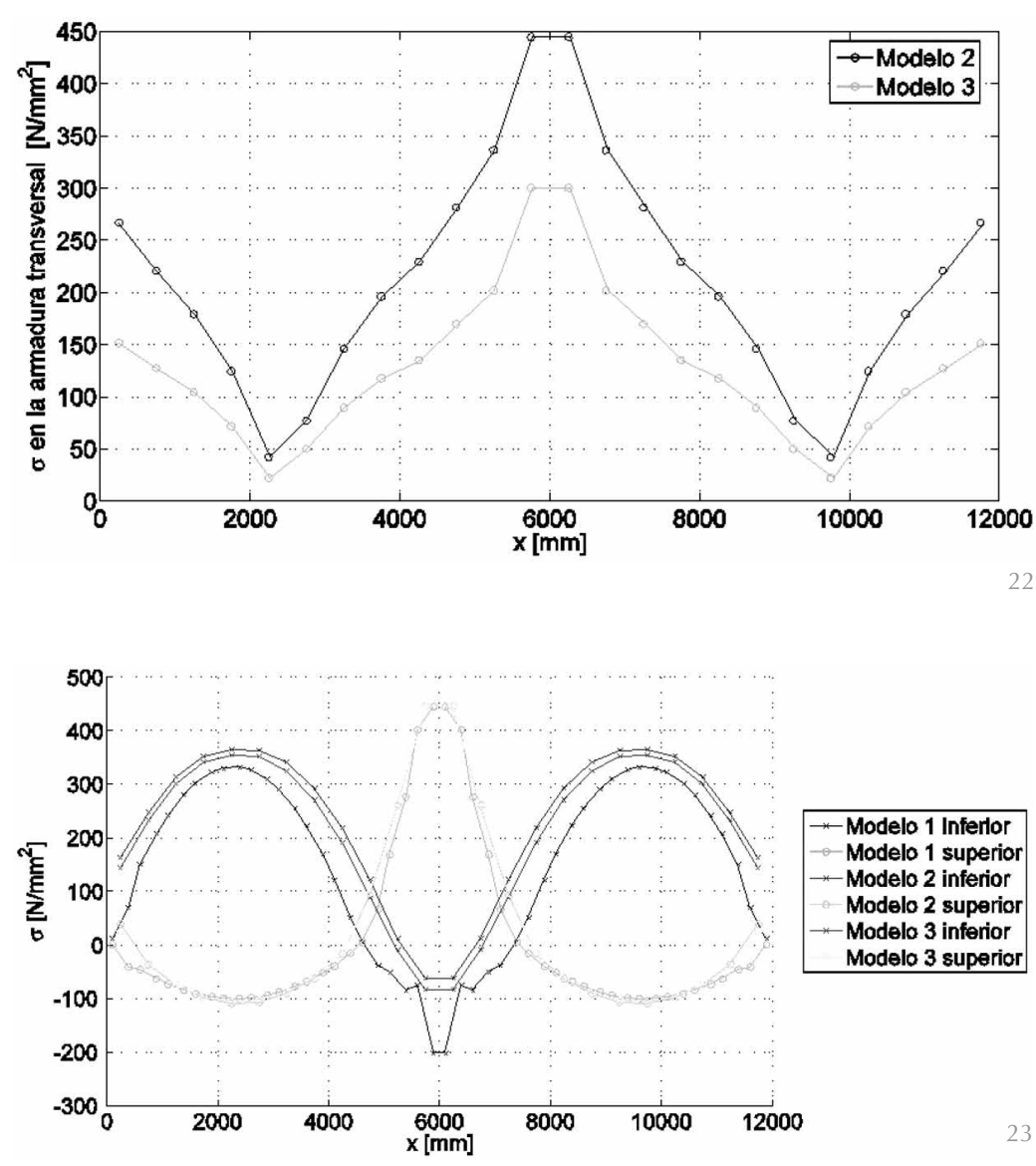

Modelo 1

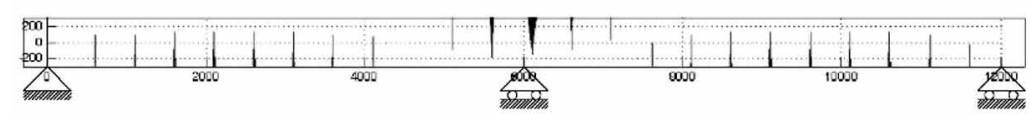

Modelo 2

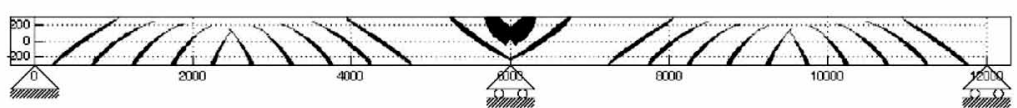

Modelo 3

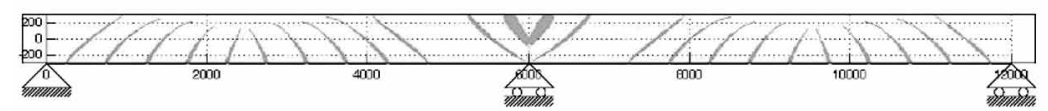

24

22. Distribución de tensiones en la armadura transversal en el centro de la sección para $\mathrm{q}=127 \mathrm{Kn} / \mathrm{m}$.

24. Patrones de fisuración para $q=127 \mathrm{Kn} / \mathrm{m}$, posterior a la primera plastificación de armadura.

23. Distribución de tensiones en la armadura longitudinal para $\mathrm{q}=127$ $\mathrm{Kn} / \mathrm{m}$ de cortante máximo. Asimismo, en la zona donde existe cortante y momento concomitante existe un incremento de tracciones en la armadura transversal que podría ser motivo de un "decalaje" de la ley de cortantes para tener en cuenta la interacción del flector en la armadura transversal. También se aprecia en esta figura que el Modelo 2 sufre plastificación de la armadura transversal en la zona del apoyo interior.

La distribución de las tensiones en las armaduras longitudinales se muestra en la Fig. 23 donde se hace evidente el incremento de tracciones en la armadura longitudinal por efecto del cortante concomitante. Por otro lado, se aprecia una mayor extensión de la rótula plástica alrededor del apoyo interior que lo observado para el modelo sin cortante.

La Fig. 24 muestra las distribuciones de las fisuras en los tres modelos para una carga en rango de servicio y en rango de estado límite último respectivamente. El grosor de las líneas de fisuras es proporcional al ancho de fisura estimado de cada modelo. Se aprecia inmediatamente que la distribución de fisuras del Modelo 1 no es adecuado para las zonas con cortante importante. Asimismo, los modelos que consideran el esfuerzo de cortante representan mejor el esquema típico de fisuras en el que la primera fisuración inclinada se prolonga en forma de arco a lo largo del eje de la viga.

En la zona del apoyo central, con esfuerzo cortante y flector importante, tiene lugar una fisuración inclinada que no es capaz de reproducir el modelo de esfuerzos normales. La inclinación de las fisuras es mayor para el Modelo 2 así como el ancho de fisura estimado. Esto se evidencia en la Fig. 24 donde se aprecia la influencia de la armadura transversal en el ancho de fisura estimado.

\section{3. interacción esfuerzos normales- torsión}

La distribución de tensiones debidas a un esfuerzo torsión depende considerablemente de la geometría de la sección y se produce, en general para secciones no circulares, alabeo de la geometría en rango elástico. Después de producirse la fisuración por torsión, la distribución de tensiones depende además de la distribución de armaduras, y se producen otros efectos cinemáticas tales como la distorsión de la sección. La Fig. 25a muestra la distribución de tensiones tangenciales antes y después de producirse la fisuración de la sección cuya geometría se muestra en la Fig. 25b. Se aprecia que tras la fisuración se produce un cambio en la distribución del flujo haciendo que se asemeje al de una sección tubular corroborando las hipótesis 
tradicionalmente asumidas por las normativas de proyecto. Cabe mencionar que este fenómeno es obtenido por el modelo como un resultado estricto de aplicar las consideraciones supuesta por el usuario.

En la Fig. 26 se muestran las distribuciones de tensiones principales de compresión para la situación no fisurada y fisurada, para el mismo nivel de carga. En la misma figura se indica la distorsión de la sección, aumentada por un factor de escala de 1000. Es evidente que la sección no fisurada no sufre distorsión apreciable, en coherencia con la teoría de elasticidad isótropa. Por otro lado, la anisotropía que tiene lugar en la sección fisurada produce la distorsión de la sección y el consiguiente alargamiento de las armaduras transversales. Es evidente que, de igual forma que las fibras localizadas en los campos de compresión concentrados en los bordes sufren una flexión localizada, constatada en algunos estudios experimentales, p.e. Onsongo (18), y que es producto de la compatibilidad de deformaciones de la distorsión. Finalmente, tiene lugar un acoplamiento de los esfuerzos de flexión y axil con la torsión tal y como se aprecia en la matriz de rigidez seccional y que se representa en la Fig. 27.

\subsection{Confinamiento de secciones de hormigón armado}

La disposición de armadura transversal en una sección, además de permitir la formación del mecanismo resistente de los esfuerzos tangenciales, en suficiente cantidad produce el confinamiento del hormigón y aumenta su resistencia y ductilidad. El mecanismo que permite esta mejora en el comportamiento del hormigón es fruto del complejo estado tridimensional de tensiones que tiene lugar en la sección transversal al interactuar la deformación lateral del hormigón con la armadura transversal.

Algunas normativas modernas incluyen formulaciones para valorar y cuantificar el confinamiento en función de la cuantía de las

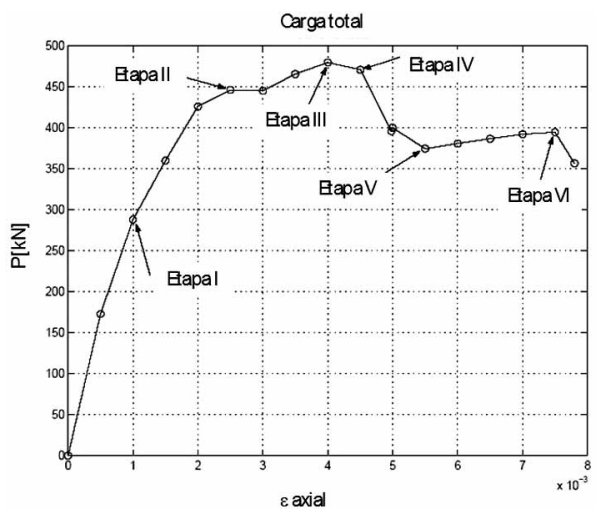

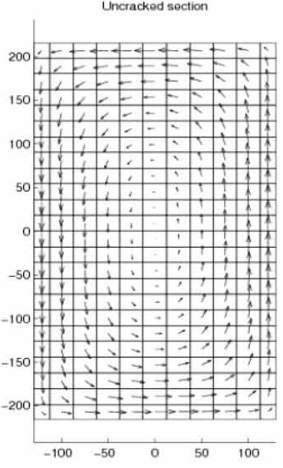
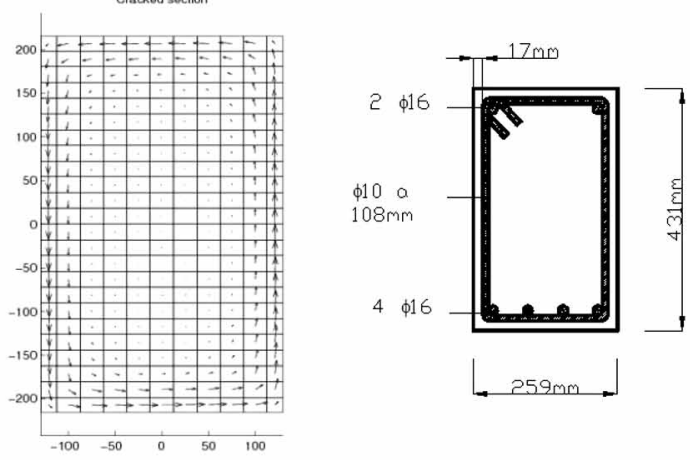

a)

b)
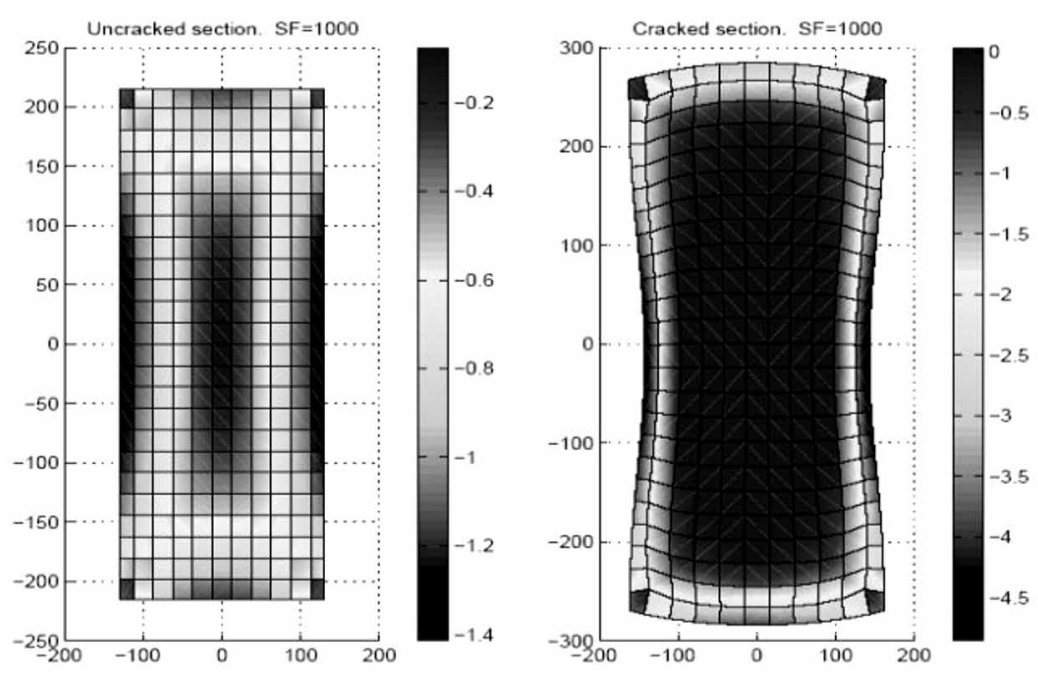

26

$\mathbf{K}_{s}=$

$\mathbf{K}_{s}=$
$\left[\begin{array}{cccccc}6.22 E 08 & -1.31 E 07 & 0 & -3.04 E 10 & -2.46 E 10 & 0 \\ -1.31 E 07 & 1.15 E 08 & 0 & 2.42 E 09 & 2.57 E 10 & 0 \\ 0 & 0 & 1.80 E 08 & 0 & 0 & 2.19 E 10 \\ -3.04 E 10 & 2.42 E 09 & 0 & 3.58 E 12 & 8.38 E 11 & 0 \\ -2.46 E 10 & 2.57 E 10 & 0 & 8.38 E 11 & 1.88 E 13 & 0 \\ 0 & 0 & 2.19 E 10 & 0 & 0 & 5.99 E 12\end{array}\right]$

armaduras y disposición de los cercos. Estas formulaciones provienen de ajustes empíricos con diferentes esquemas de armado, y su extensión a geometrías de secciones diferentes u otros esquemas de armado no siempre es clara. El modelo presentado considera el estado tridimensional de las tensiones en la sección, ya que permite discretizar el esquema de armado transversal explícitamente, por lo que constituye una herramienta de gran utilidad para evaluar el confinamiento de secciones no habituales.

La Fig. 28 presenta el resultado de una curva fuerza axil-deformación axil en una sección rectangular de hormigón de $30 \mathrm{MPa}$ de resistencia uniaxial que ha sido confinada con armadura transversal sin considerar las armaduras longitudinales. En la curva se han
25. Distribución de tensiones de cortante en una sección rectangular. a) Flujo de tensiones antes y después de fisurar. b) Sección transversal analizada.

26. Distribución de tensiones principales de compresión antes y después de fisurar y distorsión de la sección (factor de escala 1000).

27. Matriz de rigidez fisurada de la sección.

28. Curva esfuerzo axil-deformación para un pilar rectangular confinado con armadura transversal. 

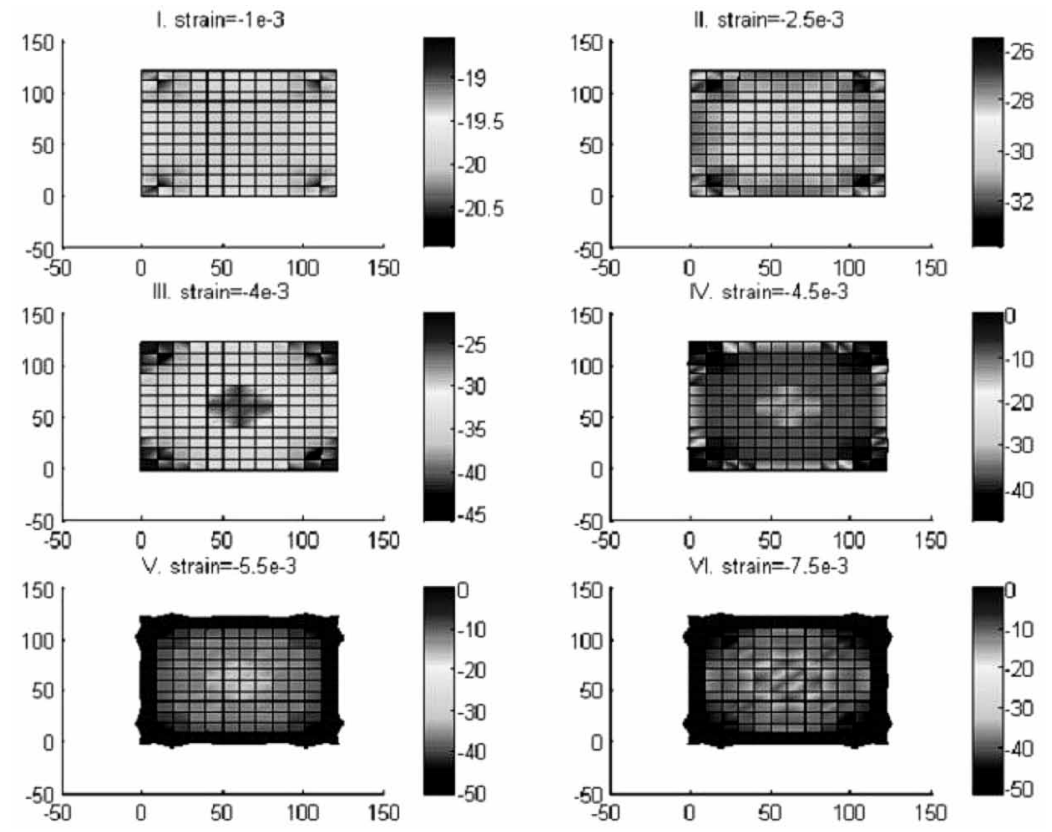

29

29. Tensiones normales y distorsión de la sección para diferentes estados de carga axil. representado seis etapas representativas del proceso de carga. Además, se muestra la distorsión de la sección para identificar las situaciones de pérdida de recubrimiento. Puede apreciarse en la etapa II la menor eficiencia del armado transversal para confinar las esquinas de la sección. El salto de recubrimiento se origina en dichos puntos tal y como se aprecia en las etapas III y IV, Fig. 29. A partir de la Etapa $V$ el recubrimiento ya se ha perdido por completo, hecho que se aprecia por la gran distorsión localizada en esa zona de la viga. Las tensiones de compresión alcanzan valores de hasta 50 MPa siendo más importante el aumento en las zonas de las esquinas interiores de la armadura transversal.

\section{CONCLUSIONES}

Los efectos tridimensionales que tienen lugar en elementos lineales de hormigón armado pueden ser muy relevantes en el comportamiento real de dichas estructuras para algunos casos de carga, especialmente cuando los esfuerzos tangenciales son importantes o se dispone de suficiente armadura como para confinar suficientemente el hormigón. Los elementos tradicionales para modelizar vigas y columnas que se basan sólo en el comportamiento del hormigón bajo esfuerzos normales pueden resultar muy útiles y satisfactorios para un gran número de casos prácticos, si bien no son adecuados en otros casos. En este artículo se ha mostrado que los modelos de flexión dominante no son capaces de reproducir fenómenos que pueden ser muy importantes como: fisuración inclinada, incrementos de tensiones en armaduras longitudinales y transversales, bajo esfuerzos tangentes, estado triaxial en la zona confinada por armadura transversal, rigidez real ante esfuerzos cortantes, etc.

Se ha presentado un modelo numérico seccional que es capaz de reproducir los fenómenos tridimensionales en secciones de geometría cualquiera y con cualquier distribución de armado. Más aún, el modelo considera explícitamente la distorsión y alabeo de las secciones, aspectos que han resultado importantes para poder tratar adecuadamente el efecto de la fisuración inclinada a nivel seccional.

Algunas teorías clásicas de diseño como son el incremento de tracciones en la armadura longitudinal debido al esfuerzo cortante o la asimilación de la sección real de hormigón a una tubular equivalente se corroboran y resultan directamente de las consideraciones anteriores.

Por otra parte, algunos aspectos duales a aquéllos, derivados de la interacción flexióncortante, como el incremento de tracciones en la armadura transversal por efecto de la flexión concomitante con el cortante, se han podido identificar con la misma teoría. Estos fenómenos no se contemplan habitualmente a nivel de proyecto y deberían evaluarse las situaciones en los que éstos pueden ser importantes para poder así considerarlos con una formulación de proyecto totalmente consistente. Por otro lado, la correcta evaluación de la interacción de esfuerzos tangentes y normales es relevante para la evaluación del comportamiento no lineal de estructuras continuas y su ductilidad real. Estos aspectos son de especial importancia para mejorar el aprovechamiento de los materiales en estado límite último tanto de solicitaciones estáticas como sísmicas.

En base a los resultados obtenidos, contrastados experimentalmente, el modelo desarrollado, que está en constante proceso de evolución y mejora por parte de los autores, deviene una herramienta de gran utilidad, por su capacidad para estudiar situaciones o casos de comportamiento poco conocidos, frente a los cuales deben hacerse hipótesis simplificadas en la ingeniería cotidiana. En este sentido, se plantean futuros desarrollos y aplicaciones del modelo orientados a estudiar el comportamiento a esfuerzo cortante de secciones de formas no rectangulares para desarrollar formulaciones de proyecto, efectos del tipo de carga en la redistribución de esfuerzos y en la resistencia a cortante en vigas continuas, mecanismo resistente de secciones construidas en varias fases (p.e., alma prefabricada y ala superior in-situ) y el elementos reparados o reforzados mediante la incorporación de materiales compuestos. 


\section{AGRADECIMIENTOS}

Los autores de este artículo desean agradecer el apoyo recibido por parte del Ministerio de
Educación y Ciencia, a través de los proyectos SARCS (BIA-2006-05614) y SEDUREC (CSD2006-00060), y del Instituto para la Promoción de Armaduras Certificadas (IPAC).

\section{BIBLIOGRAFÍA}

(1) Marí, A.R. "Numerical simulation of segmental construction of three-dimensional concrete frames", Engineering Structures, Vol. 22, No. 6, 585-596, 2000.

(2) Marí, A. R., Bairán, J.M., "Evaluación de los efectos estructurales del deterioro, reparación y refuerzo, mediante análisis no lineal evolutivo", Hormigón y Acero. Vol. 60, No. 254, pp. 51-63, 2009.

(3) Vecchio, F.J., Collins, M. P., "Predicting the response of reinforced concrete beams subjected to shear using the modified compression field theory", ACl Structural Journal, Vol. 85, No. 3, pp. 258-268, 1988.

(4) Petrangeli, M., Pinto, P.E., Ciampi. V., "Fiber element for cylic bending and shear of RC structures. I: Theory". ASCE J. Eng. Mechanics, Vol. 125, No. 9, 994-1009, 1999.

(5) Ranzo, G. "Experimental and numerical studies on the seismic performance of beam-column RC structural members subjected to high shear", Doctoral Thesis, University of Rome "La Sapienza", Rome, pp. 238, 2000.

(6) Bentz, E., "Sectional analysis of reinforced concrete members", Doctoral Thesis, University of Toronto, Toronto, pp. 184, 2000.

(7) Bairán, J.M., Marí, A.R. "Coupled model for the non-linear analysis of anisotropic sections subjected to general 3D loading. Part 1: Theoretical formulation". Computers \& Structures, Vol. 84, 2254-2263, 2006. DOI: 10.1016/j.compstruc.2006.08.036.

(8) Bairán, J.M., Marí, A.R. "Coupled model for the non-linear analysis of anisotropic sections subjected to general 3D loading. Part 2: Implementation and validation". Computers \& Structures, Vol. 84, 2264-2276, 2006. DOI: 10.1016/j.compstruc.2006.08.036.

(9) Bairán, J.M., Marí, A.R., "Multiaxial-coupled analysis of RC cross-sections subjected to combined forces", Engineering Structures, Vol. 29, 1722-1738, 2007. DOI: 10.1016/j.engstruct.2006.09.007.

(10) Bairán, J.M., Marí, A.R., "Shear-Bending-Torsion Interaction in Structural Concrete Members: A Nonlinear Sectional Approach", Arch. Comput. Methods Eng., 14:249-278, 2007. DOI: 10.1007/ s11831-007-9007-5

(11) Jiménez Montoya, P., García Meseguer, A., Morán Cabré, F., "Hormigón Armado", Gustavo Gili, DL, Barcelona, 2004.

(12) Park, R., Paulay, T., "Estructuras de concreto reforzado", Ed. Limusa, México, 1996.

(13) EN-1992-1-1. "Eurocode 2: Design of Concrete Structures- Part 1-1. General Rules and Rules for Buildings", European committee for Standarization, Decembre 2004.

(14) Comisión permanente del hormigón, "Instrucción de Hormigón Estructural EHE-08", Ministrio de Fomento, Madrid, Diciembre de 2008.

(15) Bairán, J.M., Marí, A.R., "Proyecto de estructuras de hormigón armado con aceros de alta ductilidad", Universitat Politècnica de Catalunya, 2007.

(16) Hsu, T.T., "Torsion of reinforced concrete", Van Nostrand Reinhold, New York, 515.

(17) Leonhard, F., Mönnig, E., "Estructuras de hormigón armado", Tomos 1 y 3, Ed. El Ateneo, Buenos Aires, 1990.

(18) Onsongo, W. M., "The diagonal compression field theory for reinforced concrete beams subjected to combined torsion, flexure and axial load", Doctoral Thesis, University of Toronto, Toronto, pp. 246, 1978.

(19) Collins M.P. y Lampert, P., "Redistribution of moments at cracking - The key to simpler torsion design", Analysis of Structural Systems for Torsion, ACI Publication SP-35, American Concrete Institute, Detroit, 343-383, 1972.

(20) Cladera, A., Marí, A.R., "Experimental study on high-strength concrete beams failing in shear", Engineering Structures, 10 (27), pp. 1519-1527, 2005.

(21) Bairán, J.M., "A non-linear coupled model for the analysis of reinforced concrete sections under bending, shear, torsion and axial forces", Doctoral Thesis, Universitat Politècnica de Catalunya, Barcelona, pp. 229, 2005.

(22) Kani, M., "An experimental investigation of reinforced and prestressed beams in shear", Master Thesis, University of Toronto, Toronto, 1977.

(23) Tagliano, M.,"Indagine sperimentale e modellazione del comportamento anelastico di elementi in calcestruzzo armato", Dissertazione di Dottorato, Politecnico di Torino,1998.

(24) Lloveras, M., "Influència de la deformació a tallant en bigues de formigó armat fissurades", Tesina de Especialidad, ETSICCPB, Universitat Politècnica de Catalunya, 2008. 